\title{
A Study of the Application of Image Simulation System in Clinical Teaching
}

\author{
Wang Shougang, Cui Tao* \\ Affiliated Hospital of Beihua University JiLin, China \\ E-mail: wsg97@126.om \\ ${ }^{*}$ Corresponding Author
}

Keywords: Image Simulation System; PACS; Clinical Teaching

\begin{abstract}
As one of the fastest growing and the widest ranging disciplines in the field of modern medicine, Image Simulation System (ISS) is a science that displays the form of human interior structure and functional information in its pictorial form as images and implements interventional therapy [1]. The application of ISS in clinical teaching is an important part of the reform of medical education, and it is necessary to further optimize its technical application, solve the technical problems and enhance the quality of clinical teaching.
\end{abstract}

\section{Introduction}

As one of the fastest growing and the widest ranging disciplines in the field of modern medicine, Image Simulation System (ISS) is a science that displays the form of human interior structure and functional information as images and implements interventional therapy [1]. With the extensive application of cutting-edge technologies like electronics and computer technology, medical imaging technology is growing at a fast pace. It is a long-term task to energetically study the role played by the ISS in the teaching of clinical medicine. The ISS module is built on PACS (Picture Archiving and Communication System), and its image capturing and transmission adopts the DICOM 3.0 standard with matched viewing workstation, diagnosis workstation, teaching workstation, digital camera, scanner, and so on. This paper mainly discusses the positive role played by ISS in clinical medicine applications and studies the existing problems.

\section{Problems and Insufficiencies Revolving the Traditional Clinical Teaching Method}

\subsection{Disadvantages of the traditional image-viewing-centered clinical practice and teaching}

In traditional clinical practice and teaching, most lectures are conducted by exposing students to medical images in succession on the basis of actual conditions. However, this method is very limited, in a way that it is normally impossible to ensure that every student is able to adequately observe the details of an image when many of them are being presented in a relatively short time frame, while the lecturers must also independently and continuously provide the corresponding explanations under great work pressure. In addition, in the traditional method, the storage and the use of the medical images are also critical issues because its repeated use in teaching may lead to lower image quality and affect the outcome of teaching and explanation. Hence, changing such a backward way of teaching by applying ISS to clinical teaching should be imperative at this point.

\subsection{Defects of general multimedia courseware-based clinical teaching}

This model fails to change the underlying model of image-based explanation; instead, it just upgrades data and still puts teachers at the center of the teaching activity, rather than putting students at the core of it, thus making it hard to fully motivate these students. As medical technology is progressing day by day and the society has a higher requirement for the quality of medical care, the cramming-based teaching method is already unable to substantially improve the quality of clinical teaching. Most of the frequently-used medical images for clinical teaching at present translate the existing data into images and digital form through a device, or sort and flatten the comprehensive case database, further classifying and organizing it in .jpg or .bmp formats according to the subjects and types of diseases. A lot of information unable to be displayed in the 
pictorial form are lost imperceptibly in this process, and such losses will make the cases incomprehensive when being displayed. Besides, this data sorting method is very time-consuming, and when it comes to sorting more complicated cases, one tends to neglect the particularity of these data, leading to the loss of practical significance of these data. As a result of the application drawbacks of multimedia courseware, clinical teaching needs to be further revolutionized technically and methodologically. Hence, making the utmost of the PACS-based image simulation technique to integrate multimedia resources is a vital path to enhance the quality of clinical teaching on all fronts.

\section{Main Advantages of the Application of ISS in Clinical Teaching}

\subsection{Abundant provision of image data}

The PACS and the ISS synthesize digital medical images and information into a database. Normally, various image collection devices will be linked with a high-speed computing computer as its core, and a high-capacity hard disk storage will be used to transmit, organize, and store digital information and classify these medical data by its subjects and types, thus establishing a retrieval system. Its main characteristics are high data integrity, high timeliness, high shareability, convenient access, and also robustness in terms of data-loss prevention during transmission and processing. The system has set up a complete database for information collection and records the whole process in real time when a patient enters the hospital and the treatment is initiated, while in the past model, the storage of ultrasonic image data basically needs one to copy, print, make a report manually, including the use of videotaping equipment. Moreover, some data cannot be saved without occupying some physical space, which also limits its storage life cycle, requiring these data to be manually scoured when needed for use, which is inefficient. When PACS is used, an authenticated identity may be used to quickly login to the system via an output platform and keywords can be inputted to find the related data without being limited in terms of space and time.

\subsection{The reasonable organization of clinical teaching data}

Various imagological examinations are closely interrelated as a general characteristic, and imagological examination is closely related to medical history, signs, pathological examination and examination results of patients. Clinical teaching should make comprehensive connection and comparison according to the general characteristics of the teaching contents, compare the data on similar cases, and expand the contents of related courses. Such a teaching model is unachievable through the traditional teaching methods, because it takes a lot of time to collect data for lessons while the comprehensiveness and timeliness of these data also cannot be guaranteed. These data are characterized by dynamics and tridimensional character. In the teaching of a case, one may collect data about the therapeutic methods and effects in the same period of time for horizontal comparison and make use of the large storage of the system to obtain therapies and effects at different times to help students gain an insight into the improvement of therapeutic methods and major features of the current therapeutic techniques, thus developing a systematic repertoire of knowledge. The application of the system not only provides the collection and processing of hospital data with effective solutions, but also tremendously drives the advancement of clinical teaching. Clinical teaching is no longer confined to limited courseware and data, rather, it achieves real-time synchronization between cases and data by using convenient channels to acquire resources.

\subsection{The improvement of the students' practical skills}

Clinical teaching is generally characterized by dynamic change, a diversity of factors involved, and the complex actual operation, while there are very few opportunities to conduct the actual operation even in the clinical practice and internships. Furthermore, generally, teaching relies on the analysis and discussion of data and the explanation of typical cases, and it is deficient in the development of expertise and competence. The application of image simulation techniques and the PACS can solve the problem of inadequate development of practical skills to a great degree, while the related 
contents can be more flexible and changeable, and can even break through the constraints of classrooms and transferrable to a mobile terminal. Moreover, ISS and PACS are easy to operate and have a large data storage, through which teachers may lead students to look for cases for analysis on their own and sum up their experience with their opinions and reflections, which changes the traditional lecturing model into the teaching model that focuses on guiding students to think independently. For students, other than coming to internship and attending classes, they can search for information about the cases they are interested in via PACS, complete before-class preview and after-class review, make independent diagnosis of the cases according to these information, so as to enhance their initiative in studying, diversify the learning methods, and develop their clinical thinking. This is also in accord with the mentality of the present educational reform, changes the teacher-led classroom into student-oriented education, and ultimately increases the teaching efficiency substantially.

\section{Application scheme}

\subsection{Organizing and preparing data}

Long-term collection and sorting of data on cases were implemented and a medical image viewing and diagnosis reporting system was established to make it convenient for users to quickly search the pertinent image data on cases. For example, if you search for information about tumor in internal medicine, you can enter "internal medicine" via a retrieval catalog and then enter the keyword "tumor" to inquire all kinds of images and information about tumor cases. From the information given above, it is also possible to select high-quality and extremely typical cases using the advanced filtering function by time and region.

\subsection{Comparing teaching strategies}

The application of ISS and PACS to clinical teaching makes it convenient to pinpoint cases and it allows the viewing of images according to information of patients in PACS, which offers powerful image processing functions, such as zooming in or out, definition and resolution modification, window level and window width stretching, actual length evaluation, and application of image simulation software to construct a focus model. Teachers may adjust an image according to the actual teaching requirements and demonstrate multiple cases on split screens and compare the characteristics of different focuses or display different stages of focuses, to enhance the dynamics of teaching and make it easier for students to comprehend. PACS may also be used to assess students in real time and grasp their overall learning performance. Before this system was applied, organizing a quiz would require a more complicated preparation of larger amounts of image data, which increased the workload, and rolling the films as images every time also required the spending of considerable in-class time. On the contrary, PACS allows a direct acquisition of similar cases for a quiz and allows students to independently analyze the characteristics of cases in line with the basic train of thought of the diagnoses of these cases.

\subsection{Implementing teaching schemes to increase the learning efficiency and quality of students}

In the application of ISS and PACS, teaching plans should be adjusted accordingly due to the effectiveness of the new system implementation, and two points need to be underlined in this process: the first is the high-efficiency requirement of image-viewing for the students and the second is to meet individual needs of the students. After arranging the main content of teaching, the lecturer provides the contents of clinical teaching to let students collect graphical information on their own and allows students to continue to follow and observe the treatments used in the cases according to their personal interest after the entire case have been fully explained to deepen their understanding or find other cases for analysis. This provides students with a platform to show their achievements in class, make comparisons by group, and enhance their engagement in teaching.

To sum up, the application of ISS to clinical teaching is an important part of medical education reform, and it is necessary to further optimize the technical application, solve the technical 
problems, and enhance the quality of clinical teaching.

\section{References}

[1] Li BS,Zhu DZ,Deng M.The application and advantages of PACS system in the teaching of 《Medical Imaging 》 [J].Journal of Hubei University for Nationalities (Medical Edition),2010(27).

[2] Deng DQ,Yu AL,Wei Y.Application and function of digital medical image database(mitris system)on PACS network in medical imaging practice teaching[J]. Medical Information,2011(24).

[3] Shi ZX,Zong HQ,Qin RP,et al.The role of PACS in the teaching of medical imaging specialty[J].Hebei Medical Journal,2009(31).

[4] Li MZ,Xi KM,Bao Q.Application of PACS in graduation practice examination of medical imaging[J].China Medical Devices,2009(24).

[5] Tang X,Tan W,Kw YJ,et al.Application of teaching PACS system in medical imaging experiment teaching[J].China Modern Doctor,2010(48).

[6] Tan YL,Zhao ZQ,Yang MH,et al.The application of PACS in medical imaging practice teaching[J].China Practical Medical,2010(05). 